\title{
Research Paper: Determination of the Needs of Mothering Handling Training for Family Caregiving of Children and Youth With Cerebral Palsy at Home Based on a Family- Centered Approach
}

\author{
Hamid Dalvand ${ }^{1}$, Leila Dehghan $^{1}$, Abdolreza Yavari², Azadeh Riyahi ${ }^{1}$, Akram Valizadeh ${ }^{2}$ \\ 1. Department of Occupational Therapy, School of Rehabilitation, Arak University of Medical Sciences, Arak, Iran. \\ 2. Department of Speech Therapy, School of Rehabilitation, Arak University of Medical Sciences, Arak, Iran.
}

Citation: Dalvand H, Dehghan L, Yavari AR, Riyahi A, Valizadeh A. [Determination of the Needs of Mothering Handling Training for Family Caregiving of Children and Youth With Cerebral Palsy at Home Based on a Family-Centered Approach (Persian)]. Journal of Rehabilitation. 2016; 17(3): 212-221. http://dx.doi.org/10.21859/jrehab-1703212

doi): http://dx.doi.org/10.21859/jrehab-1703212

Received: 13 Jan. 2016 Accepted: 16 Apr. 2016

Keywords: Child with cerebral palsy, Manual ability classification system, Mothering handling training

\begin{abstract}
Objective Caregivers of children with cerebral palsy (CP) are faced with many challenges in self-care, treatment follow-up, educational care, and personal leisure time. Therefore, they should be trained for resolving their needs during caregiving. This study aimed to determine the priority of needs of mothering handling training for family caregiving of children and youth with $\mathrm{CP}$ at home based on the familycentered approach.

Materials \& Methods This cross-sectional study was performed on 186 children with CP (aged 4-12 years) from the rehabilitation clinics in the city of Arak. The participants were chosen by a convenience sampling strategy. Clinical tests such as the Manual Ability Classification System (MACS) and the Canadian Occupational performance measure were used to assess the level of fine motor function and determine the priority of needs of children with $\mathrm{CP}$, respectively. The data were analyzed using statistical tests including two-way ANOVA.

Results In this study, there were 89 girls and 97 boys with $\mathrm{CP}$ with a mean age $7.52+2.38$ years. The $\mathrm{CP}$ types were as follows: spastic (67.7\%), dyskinetic (13.9\%), ataxic (5.9\%), and hypotonic (4.8\%). The most important priority of needs of mothering handling training was self-care and care related to children with $\mathrm{CP}$ in the level of $\mathrm{V}$ of MACS. The results showed that the priority of needs of the mothering handling training based on occupational performance (self-care, productivity and play/leisure) were toileting in the level of IV of MACS. There were no significant differences in the priority of needs of the training in the areas of sex and level of fine motor function ( $P>0.05)$; however, there were significant differences in age and self-care $(\mathrm{P}<0.05)$.

Conclusion The results showed that the highest priority of needs of mothering handling training was selfcare, feeding, toileting, and functional mobility. However, the needs of mothering handling training enhanced with increasing severity of fine motor function lesions and age. The simultaneous relationships between age and MACS levels in children with CP have no effect on the priorities of mothering handling training. Organized training of families and caregivers about needs of mothering handling training and proper care of their children are facilitating steps in promoting the education of mothers of children with $\mathrm{CP}$. It seems that the understanding and education of mothering handling for therapists responsible for managing children with $\mathrm{CP}$ and the education of their parents can open the way to effective caring and treatment.
\end{abstract}

\section{* Corresponding Author:}

Leila Dehghan, PhD

Address: Department of Occupational Therapy, School of Rehabilitation, Arak University of Medical Sciences, Arak, Iran.

Tel: +98 (86) 33136090

E-Mail: Idehghan@tums.ac.ir 


\title{
تعيين اولويت نيازهاى آموزش هندلينَّ مادرى به مراقبان خانوادَّى كودكان مبتلا به فلج مغزى براساس رويكرد خانوادهمحور أنيار
}

\author{
حميد دالوند'، "ليلا دهقان'، عبدالرضا ياورى'، آزاده رياحى'، اكرم ولىزاده'

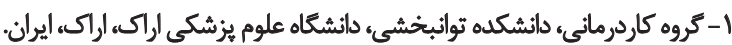

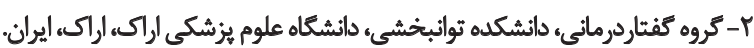

\begin{abstract}
حكيد

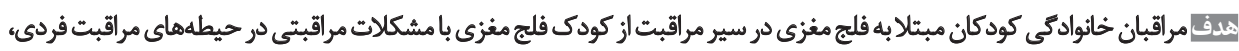

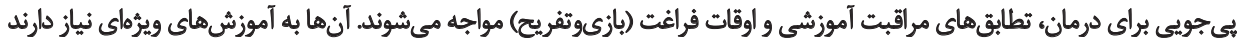

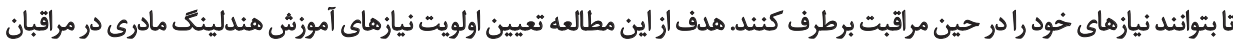

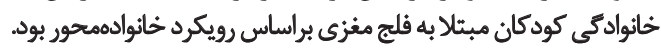

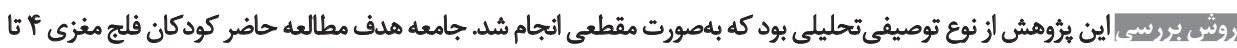

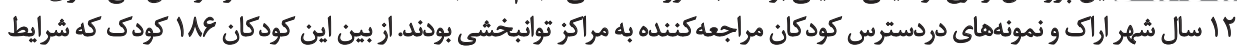

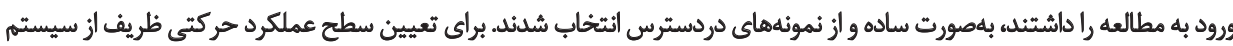

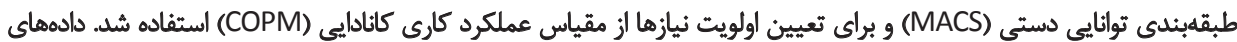

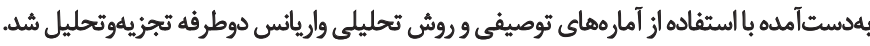

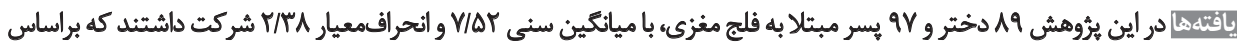

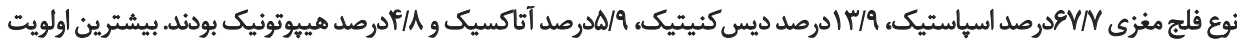

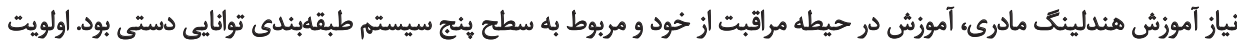

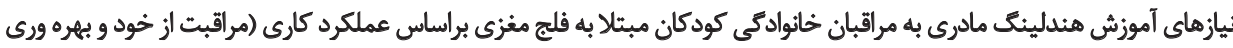

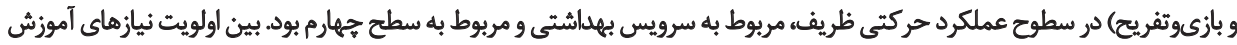

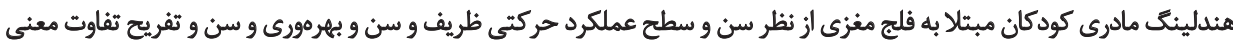

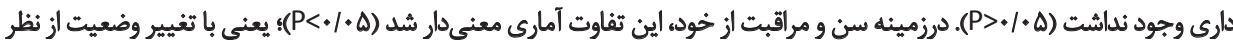

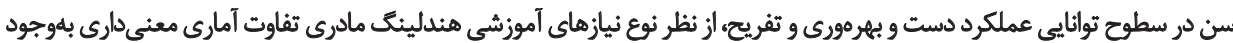

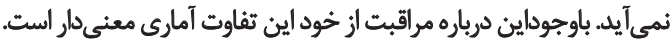

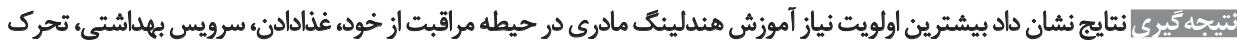

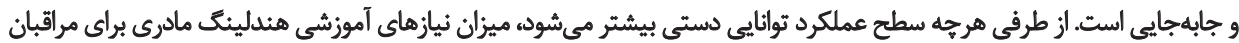

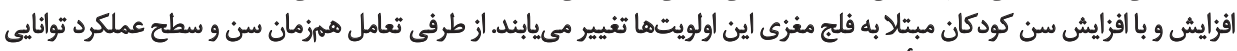

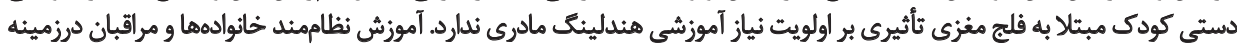

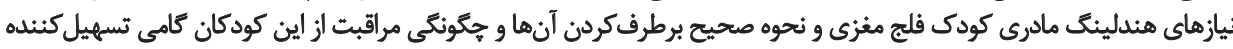

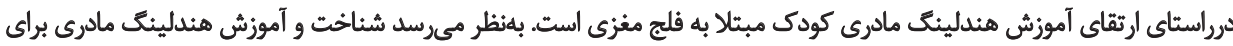

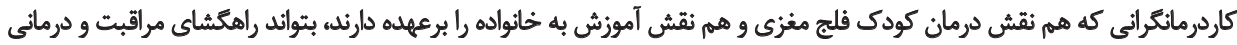

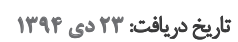

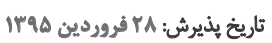


و والدين آنها از نظر اولويتهاى خانواده نشان داد كه اولويت مقدمه

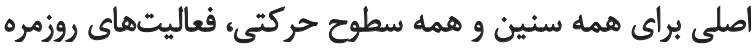

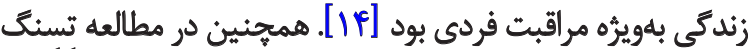

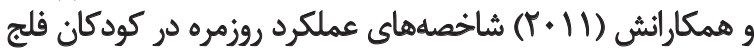

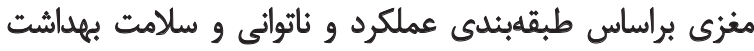

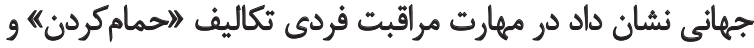

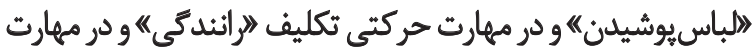

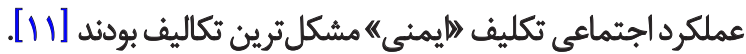

نتايج مطالعهاى توصيفى بلمنظور ارزيابى نيازهاى كودكان

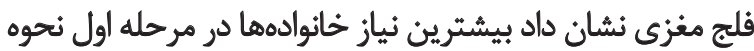

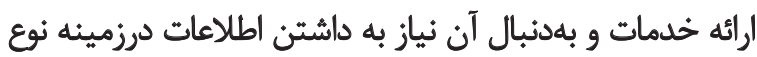

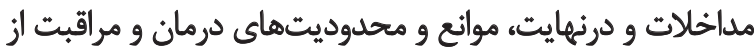

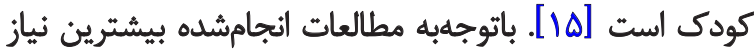

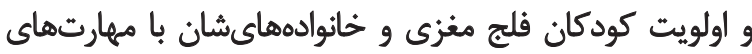

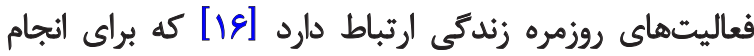

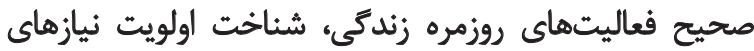

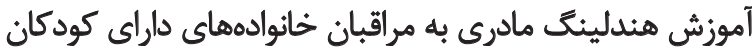

$$
\text { فلج مغزى ضرورى است. }
$$

هندلينَ مادرى نوعى مراقبت ويرٔه از كودى فلج مغزى است

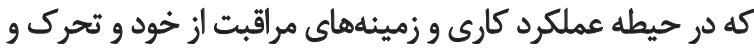

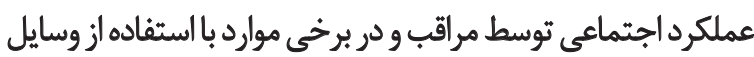

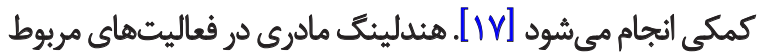

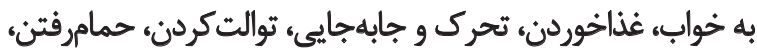

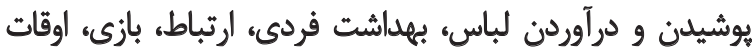

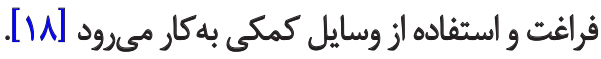

بررسى جامعى از اولويت نيازهاى آموزشى هندلينت مادرى

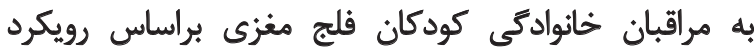

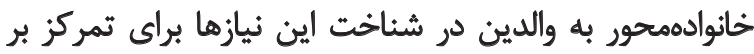

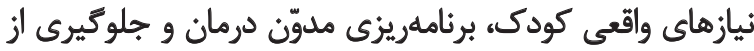

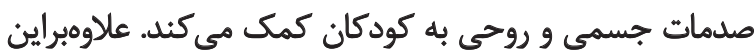

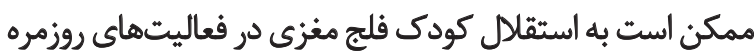

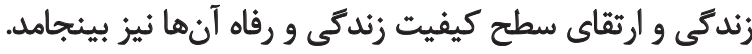

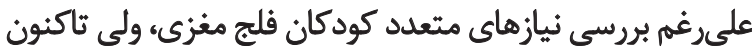

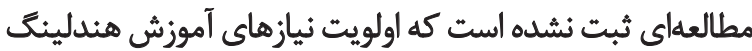

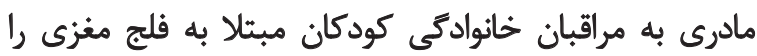

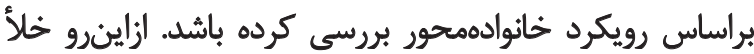

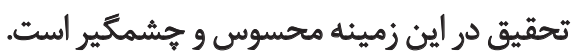

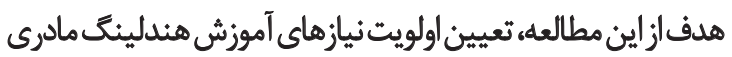

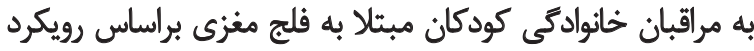

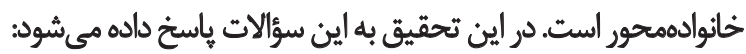
إ. اولويت نيازهاى آموزش هندلينَ مادرى كودكان مبتلابه فلج مغزى براساس سيستم طبقابئدى توانيايى دستى كداماند؟

مراقبان كودكان فلج مغزى در سير مراقبت از كودى فلج مغزى مرئ

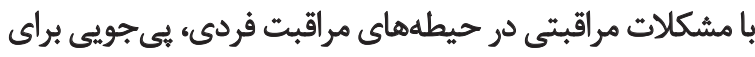

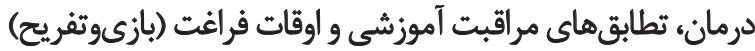

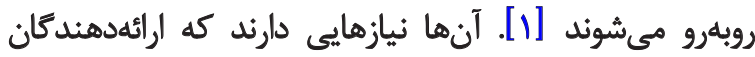

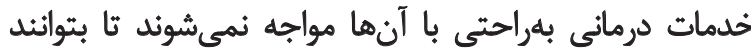

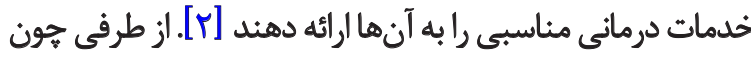
مشكل كودكان فلج مغزى طولانيى مئى نيازهاى كودكان فلج مغزى هم سير ادامهدارى خواهد دانه داشت.

بهنظر مىرسد بهتر است نيازهاى كودكان فلج مغزى و ورئو

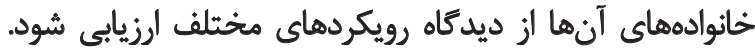

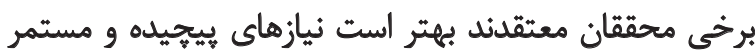

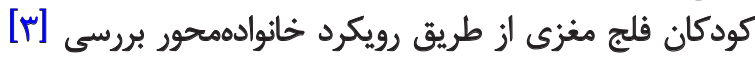

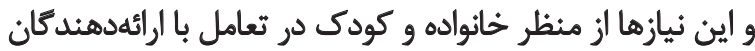

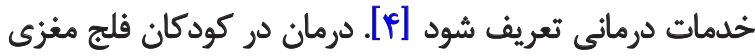

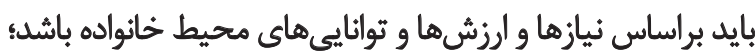

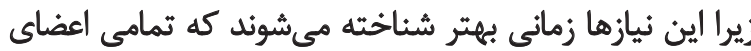

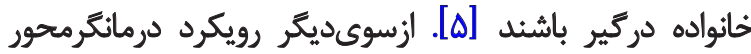

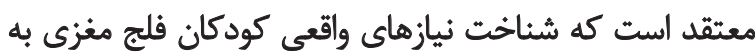

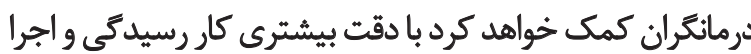

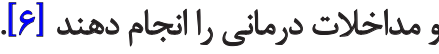

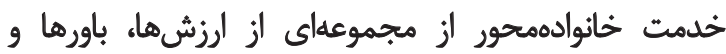

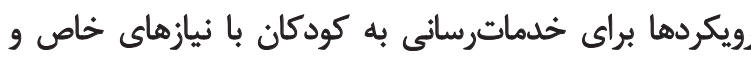

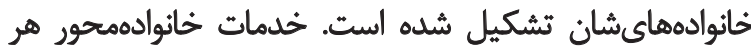

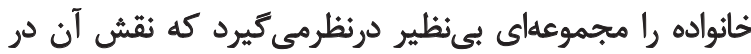

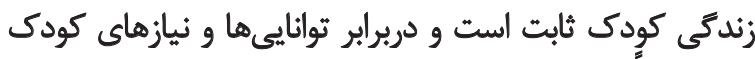

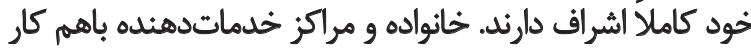

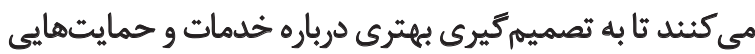

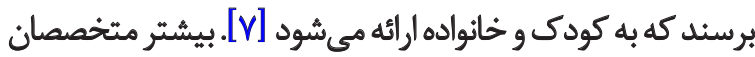

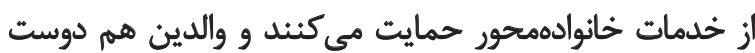

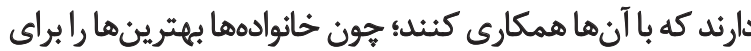

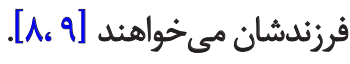

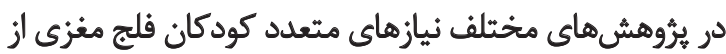

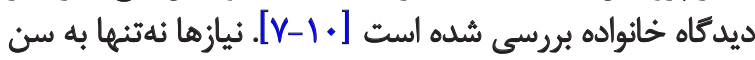

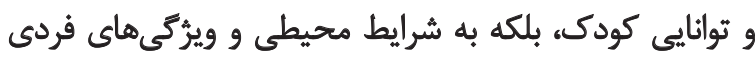

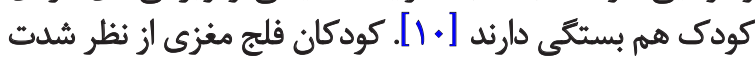

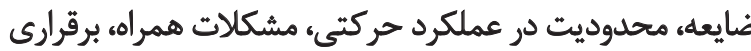

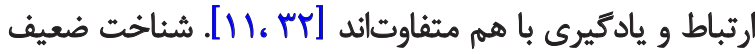

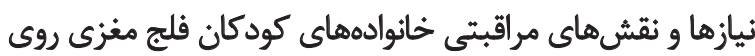

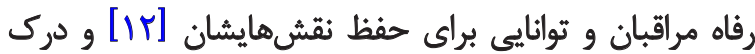

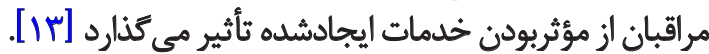

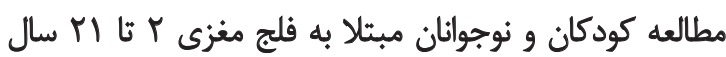


يعنى يا موقعيت از قبل آماده شده باشد يا از وسايل كمكى براى

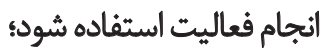

ه. كودى نمى تواند اشيا را كنترل كئد و توانايى كمى در انجام

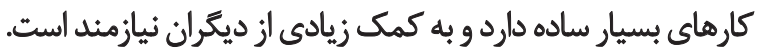
نسخه اصلى سيستم طبقهبندى توانايى دست به زبان فارسى تإسى

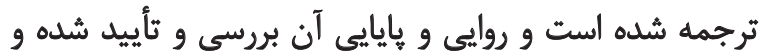

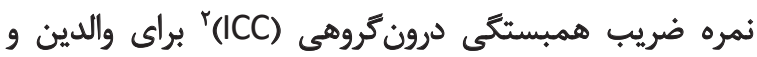

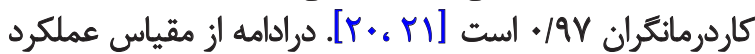

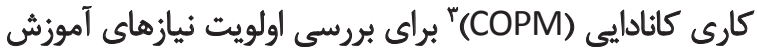

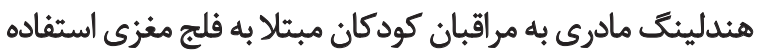

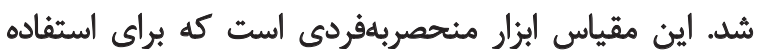

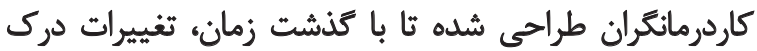

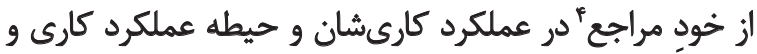

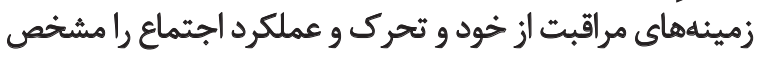

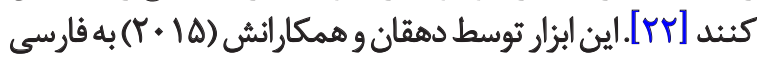

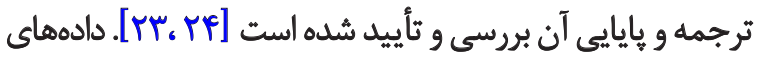

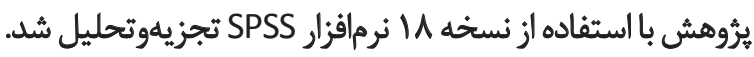

يافتهها

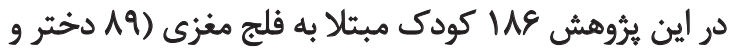

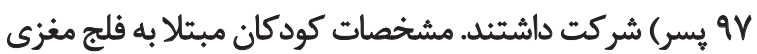

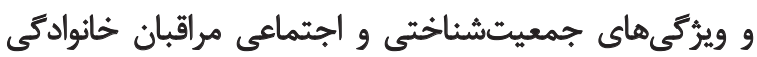

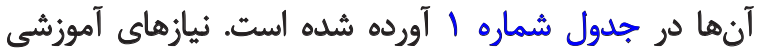

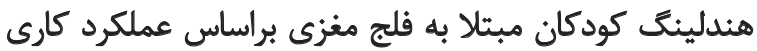

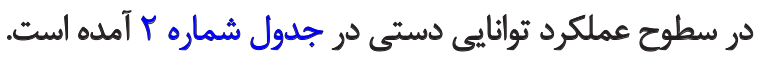

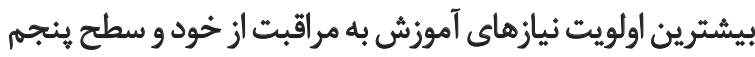

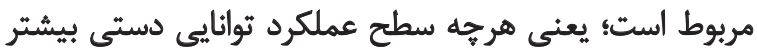

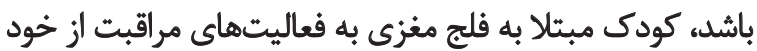
بيشترى از طرف مراقبان خانوادكى نياز دارد.

اولويت نيازهاى آموزش هندلينگ مادرى به مراقبان خانوادكى

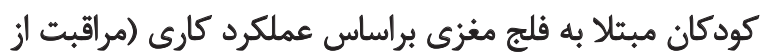

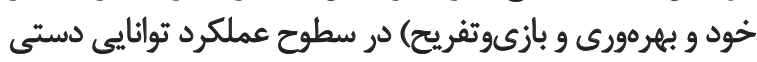

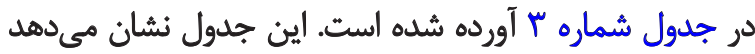

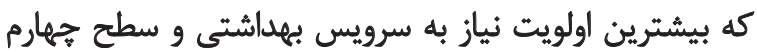

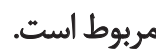

در جدول شماره F با استفاده از تحليل واريانس دوطرفه

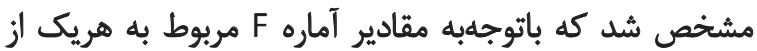

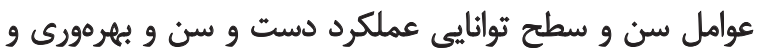

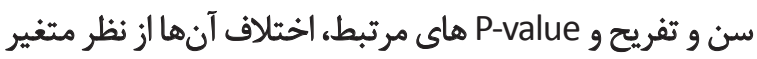

2. Interclass Correlation Coefficient (ICC)

3. Canadian Occupational Performance Measure (COPM)

4. Self-client r. آيا تفاوتى بين اولويت نيازهاى آموزش هيندلينك مادرى

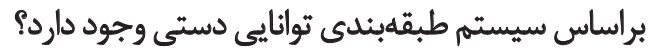

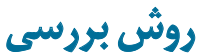

اين يُروهش از نوع مطالعه توصيفى تحليلى بود كه بلهصورت

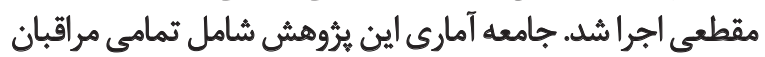

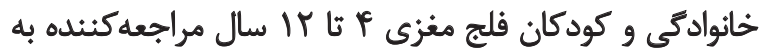

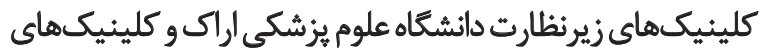

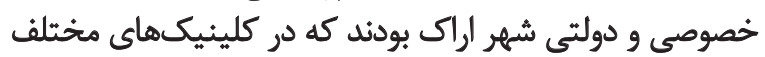

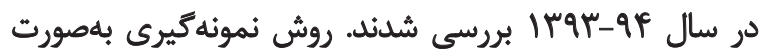

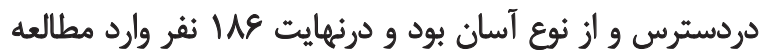

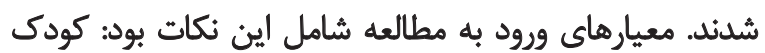

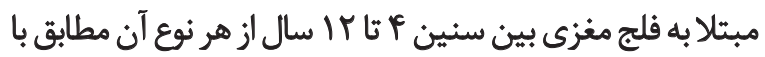

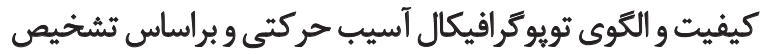

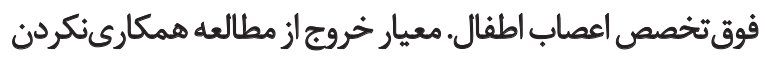
مراقبان و كودكان در هر مرحله از انجام تحقيق بودا

ووش اجرا

از بين مراقبان خانوادكى و كودكان مبتلا به فلج مغزي كه براي

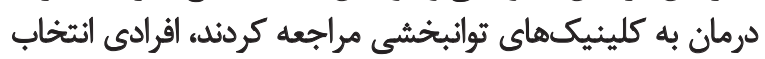

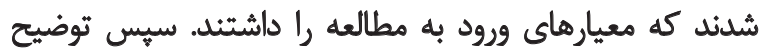

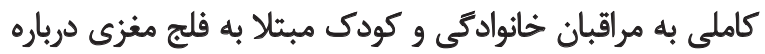

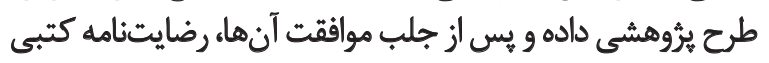

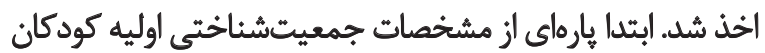

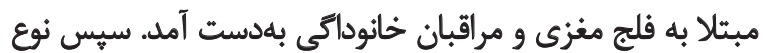

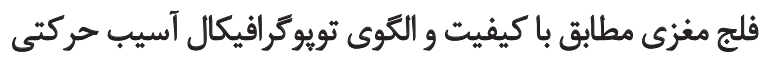

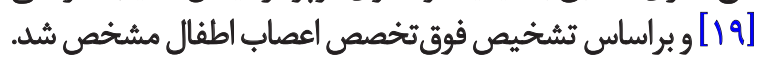

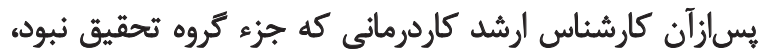

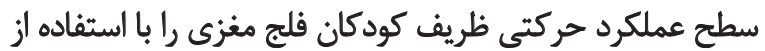

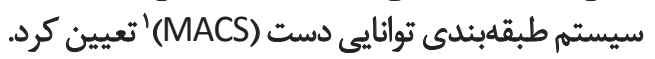

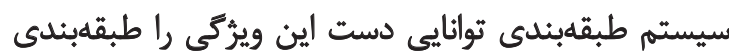

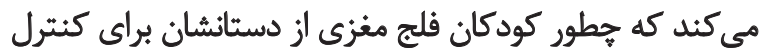

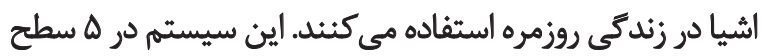
تعريف مي شود. اين سطوح عبارت است:

1. كودى اشيا را بهراحتى و با موفقيت كنترل مى كند؛

r. كودك بيشتر اشيا را كنترل مى كند؛ اما تا اندازماى كيفيت

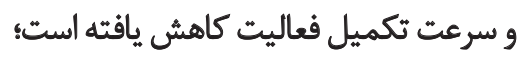

با. كودى اشيا را بهاستتى كنترل مي كند و براي آمادهكردن و يا ياصلاح در انجام فعاليتها به كمك نياز دارد؛ f. كودى فقط مىتواند كارهاى خيلى ساده را انجام دهد؛

1. Manual Ability Classification System (MACS) 


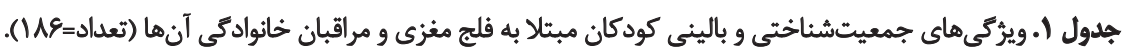

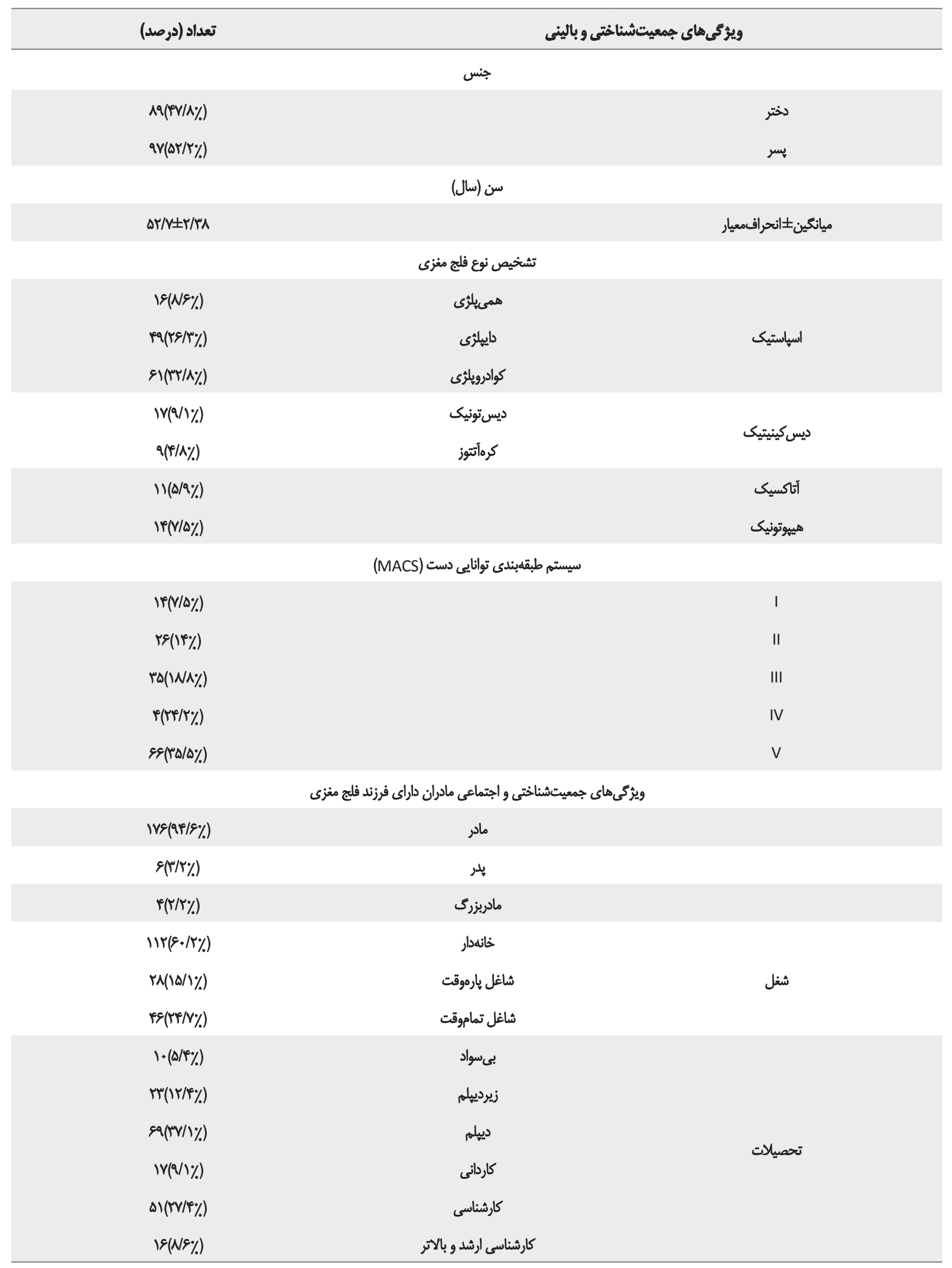

توانبخننى 
جدول ז. اولويت نيازهاى آموزش هئلينَّ مادرى كودكان مبتلا به فلج مغزى براساس عملكرد كارى در سطوح عملكرد توانايى دستى.

\begin{tabular}{|c|c|c|c|c|c|c|c|c|c|c|}
\hline \multicolumn{10}{|c|}{ سيستم طبقهبندى توانايي دستى } & \multirow{3}{*}{ عملكرد كارى } \\
\hline \multicolumn{2}{|c|}{$\mathbf{v}$} & \multicolumn{2}{|c|}{ IV } & \multicolumn{2}{|c|}{ III } & \multicolumn{2}{|c|}{ II } & \multicolumn{2}{|c|}{$\mathbf{I}$} & \\
\hline مروصد & ت تعداد & مرصد & تعداد & درصد & ت تعداد & مرحد & تعداد & ل درصد & ت تعداد & \\
\hline$r \cdot 19$ & $\Delta$ & $\pi / A$ & 18 & if & re & $\mid 0 / 8$ & rq & $\checkmark$ & ir & مراقبت از خُود \\
\hline$r / T$ & r & .10 & 1 & - & - & .10 & 1 & +10 & 1 & بهرsوى \\
\hline .10 & 1 & $1 / 8$ & $r$ & .10 & 1 & $M$ & r &.$/ \Delta$ & 1 & تثريع \\
\hline$(M T / \% \%)$ & ar & $(r \& / 9 \%)$ & Q. & $(11 \% / 0 \%)$ & rV & $(\mid Y / r \%)$ & $\pi$ & $(N) \%)$ & 10 & 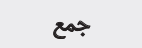 \\
\hline
\end{tabular}

توانبخنتى

جدول "ه اولويت نيازهاى آموزش هندليئك مادرى هراقبان خُانوادكى كودكان مبتلا به فلج مغزى براساس عملكرد كارى (مراقبت از خُود و بهرهورى و بازىوتفريح) در سطوح عملكرد توانايى دست.

\begin{tabular}{|c|c|c|c|c|c|c|c|}
\hline \multicolumn{6}{|c|}{ سطوح عملكرد توانايى دستى براساس MACS } & \multirow{2}{*}{\multicolumn{2}{|c|}{ أولويت نيازهاي فعاليت و مثاركت در مادرى }} \\
\hline جمع & $\mathbf{v}$ & IV & III & ॥ & $\mathbf{I}$ & & \\
\hline $\mid r(N / \% \%)$ & $\Delta(r / V \%)$ & $\Delta(T N \%)$ & $1(\Delta \%)$ & $1(\Delta \%)$ & $1(\Delta \%)$ & خواب & \multirow{6}{*}{ مراقبت از خود } \\
\hline$P r(19 / 9 v \%)$ & $\mid Q(N) \%)$ & $\Pi(ه / \uparrow \%)$ & $e(\% / \% \%)$ & $f(T / T \%)$ & $r(V / \& \%)$ & غ غنأهوردن & \\
\hline $\operatorname{er}(\pi / 9 \%)$ & $\mathbb{T}(8 / 0 \%)$ & $r(11 / \% \%)$ & $\|(\Delta / 9 \%)$ & $\mid E(N E \%)$ & $r(V / \% \%)$ & 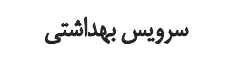 & \\
\hline $\mid \&(N \& \%)$ & $\vee(r / \Lambda \%)$ & $r(1 / 8 \%)$ & $r(V / \% \%)$ & $r(/ / / \%)$ & $1(\Delta \%)$ & حمامكردن & \\
\hline $19(1 \cdot / r \%)$ & $A(\% / \% \%)$ & $\Delta(T N \%)$ & $r(\vee / / \%)$ & $r(1 / 1 \%)$ & $r(M / / \%)$ & ميوشيلن و درأوردن لباس & \\
\hline$M(1 Y / T \%)$ & $1+(\Delta / \kappa \%)$ & $1(\Delta \%)$ & $\Delta(\tau N \%)$ & $R(T / r \%)$ & $r(V / 8 \%)$ & تحرى و جابهجايي & \\
\hline$\Delta(r / V \%)$ & $1(\Delta \%)$ & $r(\backslash / \%)$ & - & $v(\Delta \%)$ & $1(\Delta \%)$ & بازى كردن & \multirow[b]{2}{*}{ بهرهورى } \\
\hline$r(1 / 8 \%)$ & $r(v / \% \%)$ & $1(\Delta \%)$ & $\longrightarrow$ & $1(\omega \%)$ & $1(\Delta \%)$ & تكاليف ملرسه & \\
\hline$r(1 / \% \%)$ & - & $1(\Delta \%)$ & $1(\Delta \%)$ & $1(\Delta \%)$ & - & تفريع & \multirow[b]{2}{*}{ تثريح } \\
\hline $1(\cdot 10 \%)$ & $1(\Delta \%)$ & - & - & - & $\longrightarrow$ & تعاهلات اجتماعى & \\
\hline$w=(1.0 \%)$ & sT(M/K\%) & $\Delta .(N \& / 9 \%)$ & $\operatorname{TV}(\mid \mathrm{H} / \Delta \%)$ & $M(I V / T \%)$ & $1 Q(N \mid \%)$ & جمع & \\
\hline
\end{tabular}

توانبخننى

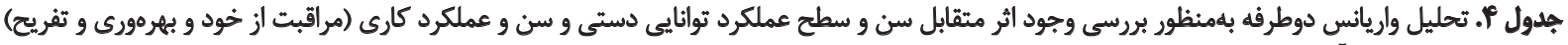

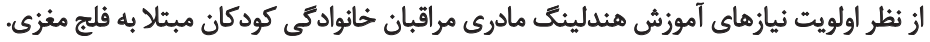

\begin{tabular}{|c|c|c|c|c|}
\hline سطح معنى دارى & درجه أزادى & مقدار أماره F & شاخصهاى أمارى & مثثيرها \\
\hline.$/ 9 \cdot 7$ & r & $.1+98$ & & سن و سطح توانايى عملكرد دست \\
\hline $.1 .+1$ & r &.$/ 900$ & سن و مراقبث ازئ خود & \\
\hline.$/ M T \Delta$ & r & ./9Vo & سن و بهره ورى & سن و عملكرد كارى \\
\hline . $/$ YTA & r &.$/ T V$ & سن و تقريح & \\
\hline
\end{tabular}


ميزان نياز خانواده تأثير دارد و خانوادههايى كه كودكانى با ضايعه

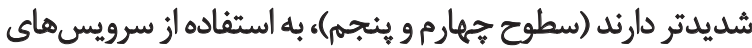

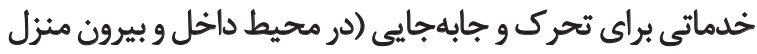

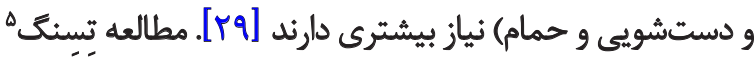

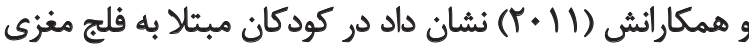

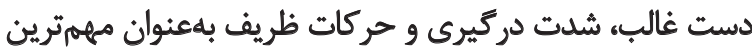

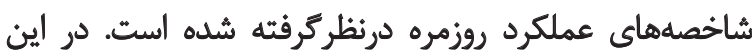

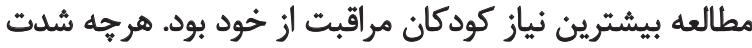

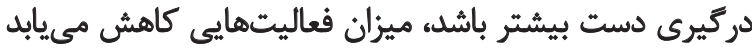

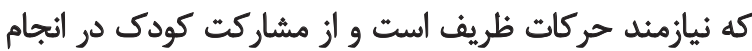

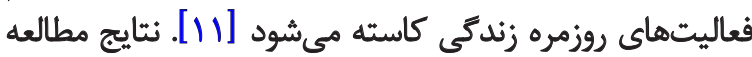
اشارهشده با نتايج مطالعه حاضر مطابقت دارد.

نتايج بهدستآمده از يُروهش حاضر نشان داد كه اولويت

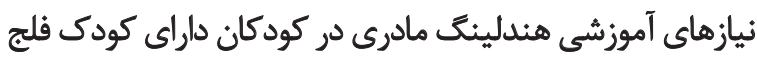

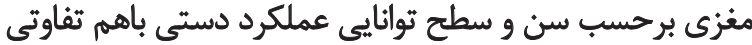

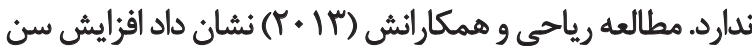

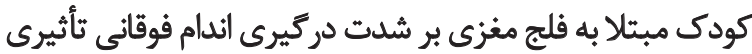

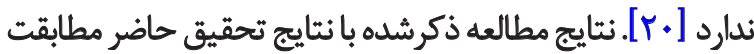

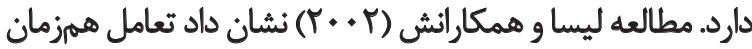

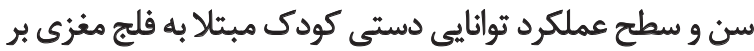

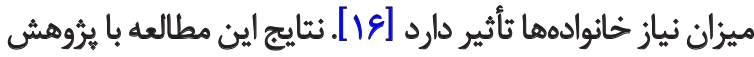

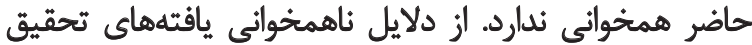

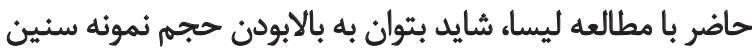

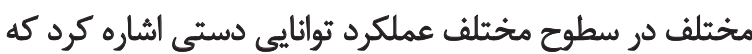
توانسته نتايج بهترى را الرائه كند.

نتايج بهدستآمده از مطالعه حاضر نشان داد اولويت نيازهاى

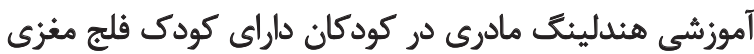

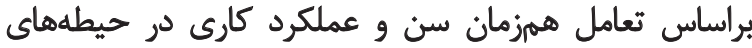

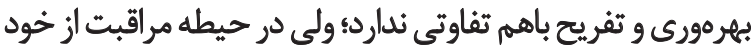

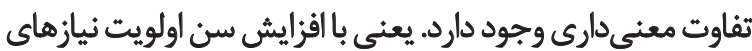

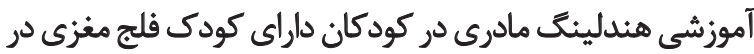

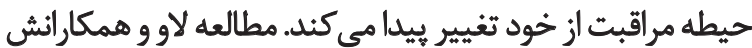

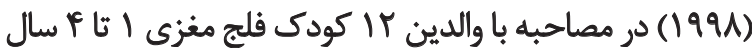

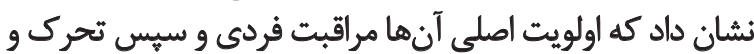

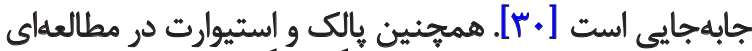

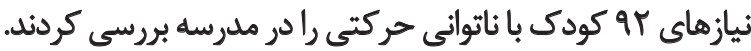

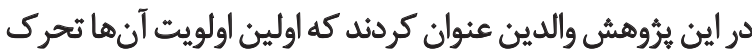

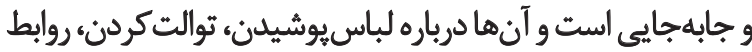

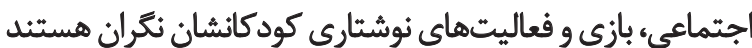

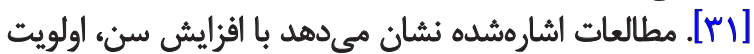

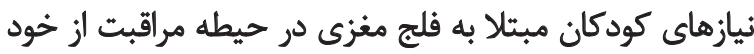

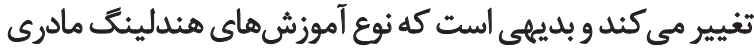

نيازهاى آموزشى هندلينك مادرى به لحاظ آمارى معنى دار نشده

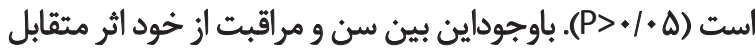

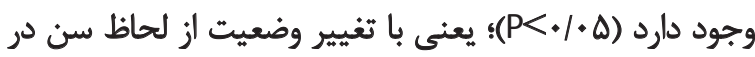

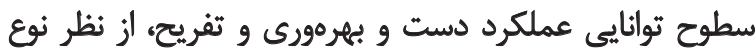

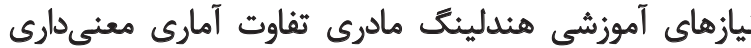

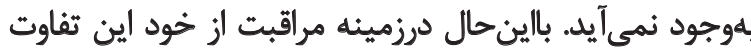

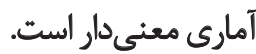

بحث

نتايج بهدستآمده از مطالعه نشان داد كه بيشترين اولويت نياز

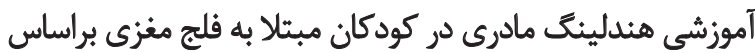

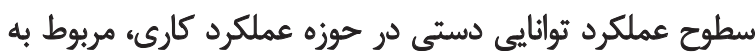

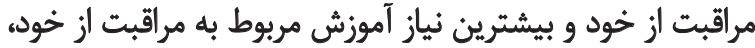

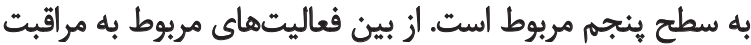

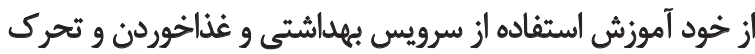

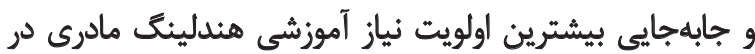

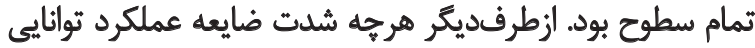

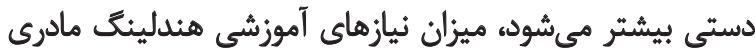

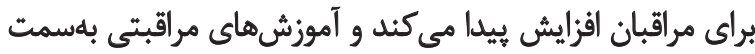

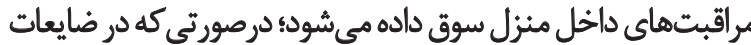

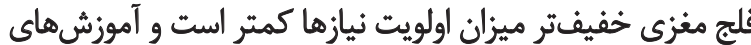

هندليتً مادرى به آموزش فعاليتهاي بيرون منزل مئل تمايل دارد.

مطالعه وركرك و همكارانش (צ • • (Y) نشان داد والدين كودكان

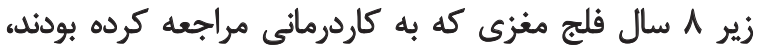

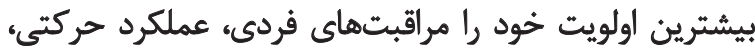

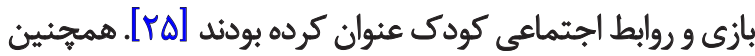

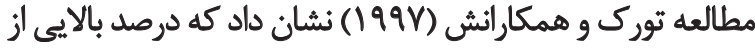

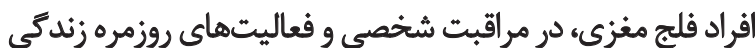

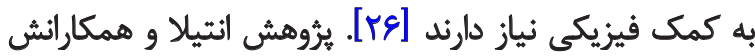

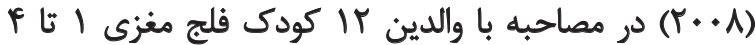

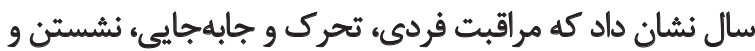

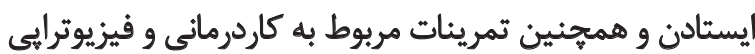

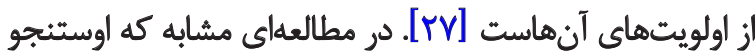

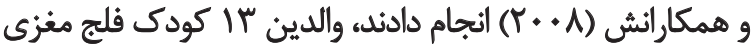

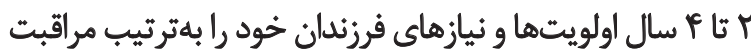

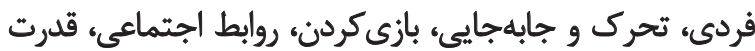

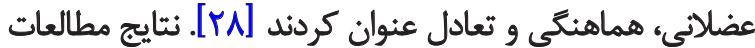

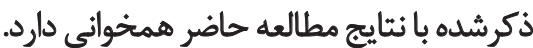

نتايج بهدست آمده از مطالعه حاضر نشان داد كه بيشترين نياز

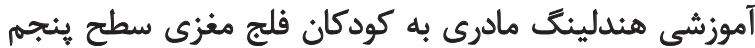

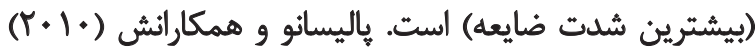

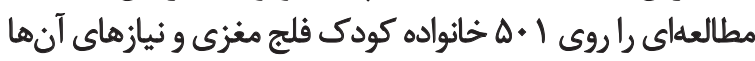

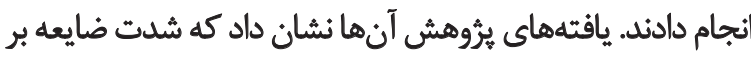




\section{References}

[1] Dalvand H, Rassafiani M, Hosseini SA, Khankeh HR. [Co-occupations: an open window to the world of caring of children with cerebral palsy (Persian)]. Journal of Rehabilitation. 2014; 14(6):8-17.

[2] McGavin H. Planning Rehabilitation. Physical \& Occupational Therapy in Pediatrics. 1998; 18(1):69-82. doi: 10.1300/ j006v18n01_05

[3] Cans C, De La Cruz J, MA M. Epidemiology of cerebral palsy. Paediatrics and Child Health. 2008; 18(9):393-8. doi: 10.1016/j. paed.2008.05.015

[4] King S, Teplicky R, King G, Rosenbaum PL. Family-centered service for children with cerebral palsy and their families: a review of the literature. Seminar Pediatric Neurology. 2004; 11(1):78-86. 10.1016/j.spen.2004.01.009

[5] Buran CF, Sawin K, Grayson P, Criss S. Family needs assessment in cerebral palsy clinic. Journal of Special Pediatric Nursing. 2009; 14(2):86-93. doi: 10.1111/j.1744-6155.2008.00176.x

[6] Rosenbaum P. Cerebral palsy: What parents and doctors want to know. British Medical Journal. 2003; 326(7396):970-4. doi: 10.1136/bmj.326.7396.970

[7] Dalvand H, Rassafiani M, Bagheri H. [Family centered approach: a literature the review (Persian)]. Modern Rehabilitation. 2014; $8(1): 1-9$.

[8] Mohamadian F, Sourtiji H, Hosseini SMS. [A new approach in rehabilitation of children with cerebral palsy (Persian)]. Journal of Research in Rehabilitation Sciences. 2012; 7(5):758-65.

[9] Law M, Rosenbaum P, King G. Family-centred service sheets: 18 educational materials designed for parents, service providers, and organizations. Hamilton, ON, Canada, McMaster University, Canadian Child Centre for Childhood Disability Research; 2003.

[10] Newey C. Improving care for children with cerebral palsy. Journal of Paediatric Nursing. 2008; 20(7):20-23. doi: 10.7748/ paed.20.7.20.s20

[11] Tseng MH, Chen KL, Shieh JY, Lu L, Huang CY. The determinants of daily function in children with cerebral palsy. Research in Developmental Disabilities. 2004; 32(1):235-45. doi: 10.1016/j. ridd.2010.09.024

[12] Kersten P, McLellan L, George S, Mullee MA, Smith JAE. Needs of carers of severely disabled people: Are they identified and met adequately? Health and Social Care in the Community. 2001; 9(4):235-43. doi: 10.1046/j.1365-2524.2001.00297.x

[13] Grant G, Nolan M, Keady J. Supporting families over the life course: Mapping temporality. Journal of Intellectual Disability Research. 2003; 47(4-5):342-51. doi: 10.1046/j.13652788.2003.00495.x

[14] Chiarello LA, Palisano RJ, Maggs JM, Orlin MN, Almasri N, Kang LJ, et al. Family priorities for activity and participation of children and youth with cerebral palsy. Physical Therapy. 2010; 90(9):1254-64. doi: 10.2522/ptj.20090388

$$
\text { هم بايد متناسب باسن آنها تغيير كند. }
$$

بيشترين نياز آموزشى هندلينگ مادرى در كودكان مبتلا به

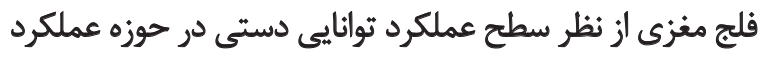

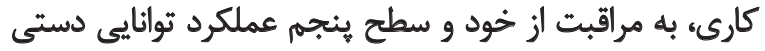

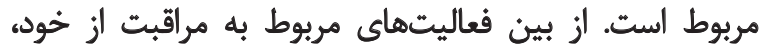

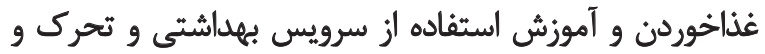

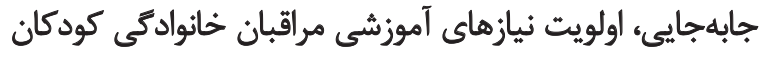

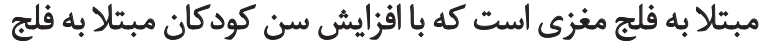

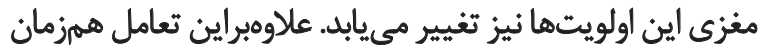

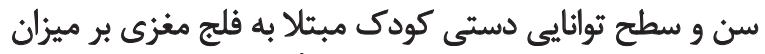

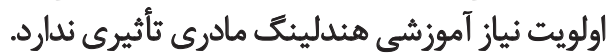

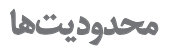

دسترسى به كودكان مبتلا به فلج مغزى و خانوادههاى آنها

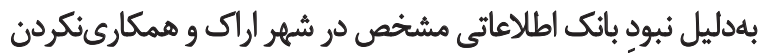
برخى ازز مراكز خصوصى توانبخشى از محدوديتهائ اين مطالعه بودي

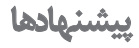
آموزش نظاممندخانوادههاو مراقبان درزمينه نيازهاى هندلينك

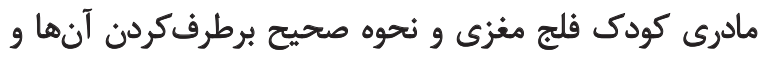

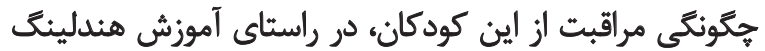

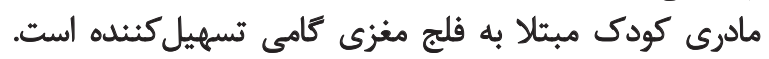

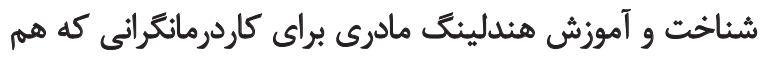

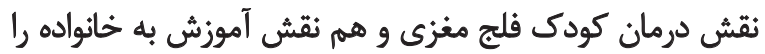

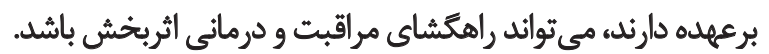

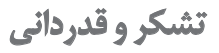

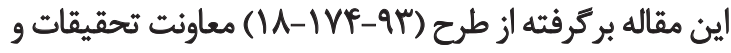

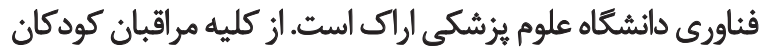

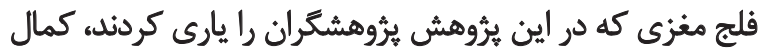

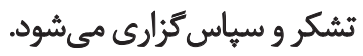


[15] Buran CF, Sawin K, Grayson P, Criss S. Family needs assessment in cerebral palsy clinic. Journal for Specialists in Pediatric Nursing. 2009; 14(2):86-93. doi: 10.1111/j.1744-6155.2008.00176.x

[16] Lisa AC, Robert J, Palisano, Jill MM, Margo NO, Almasri N, Kang LJ, et al. Family priorities for activity and participation of children and youth with cerebral palsy. Physical Therapy. 2010; 90(9):1254-64. doi: 10.2522/pti.20090388

[17] Dalvand H, Rassafiani M, Hosseini SA. [Handling in the children with cerebral palsy: a review of ideas and practices (Persian)]. Journal of Rehabilitation. 2013; 13(5):8-17.

[18] Dalvand H. [A critique of handling training (care of children with cerebral palsy): letter to editor (Persian)]. Journal of Research in Rehabilitation Sciences. 2013, 8(6):1-3.

[19] Nijhuis BJG, Reinders-Messelink HA, de Blécourt ACE, Boonstra AM, Calamé EHM, Groothoff JW, et al. Goal setting in Dutch paediatric rehabilitation. Are the needs and principal problems of children with cerebral palsy integrated into their rehabilitation goals? Clinical Rehabilitation. 2008; 22(4):348-63. doi: 10.1177/0269215507083055

[20] Riyahi A, Rassafiani M, Akbar Fahimi N, Sahaf R, Yazdani F. Cross-cultural validation of the Persian version of the Manual Ability Classification System for children with cerebral palsy. International Journal of Therapy and Rehabilitation. 2013; 20(1):1924. doi: $10.12968 /$ ijtr.2013.20.1.19

[21] Riyahi A, Rasafiyani M, Akbar Fahimi N, Karimlou M. [Reliability of the Persian Version of Manual Ability Classification System (MACS) Between Parents and Therapists in Children with Cerebral Palsy (Persian)]. Journal of Rehabilitation. 2012; 12:14-21.

[22] Law M, Baptiste S, Carswell A, McColl M, Polatajko H, Pollock N. Canadian occupational performance measure. $3^{\text {rd }}$ ed. Ottawa, Canada: CAOT Publications; 1998.

[23] Dehghan L, Dalvand H, Pourshahbaz A, Samadi SA. [Designing supplement form of the Canadian Occupational Performance Measure: item analysis and suggestions for refinement (Persian)]. Journal of Rehabilitation. 2014; 15(1):21-8.

[24] Dehghan L, Dalvand H, Pourshahbaz A. [Translation of Canadian occupational performance measure and testing Persian version validity and reliability among Iranian mothers of children with cerebral palsy (Persian)]. Modern Rehabilitation. 2015; 9(4):25-31.

[25] Verkerk GJ, Wolf MJ, Louwers AM, Meester-Delver A, Nollet $\mathrm{F}$. The reproducibility and validity of the Canadian Occupational Performance Measure in parents of children with disabilities. Clinical Rehabilitation. 2006; 20(11):980-8. doi: 10.1177/0269215506070703

[26] Turk MA, Geremski CA, Rosenbaum PF, Weber R. The health status of women with cerebral palsy. Archives of Physical Medicine and Rehabilitation. 1997; 78(12):10-17. doi: 10.1016/s00039993(97)90216-1

[27] Anttila H, Autti-Rämö I, Suoranta J, Mäkelä M, Malmivaara A. Effectiveness of physical therapy interventions for children with cerebral palsy: a systematic review. BMC Pediatrics. 2008; 8(1):14. doi: 10.1186/1471-2431-8-14
[28] ØstensjØ S, Øien I, Fallang BØ. Goal-oriented rehabilitation of preschoolers with cerebral palsy-a multi-case study of combined use of the Canadian Occupational Performance Measure (COPM) and the Goal Attainment Scaling (GAS). Developmental Neurorehabilitation. 2008; 11(4):252-9. doi: 10.1080/17518420802525500

[29] Palisano RJ, Almarsi N, Chiarello LA, Orlin MN, Bagley A, Maggs J. Family needs of parents of children and youth with cerebral palsy. Child: Care, Health and Development. 2010; 36(1):8592. doi: 10.1111/j.1365-2214.2009.01030.x

[30] Law M, Darrah J, Pollock N, King G, Rosenbaum P, Russell D. Family centered functional therapy for children with cerebral palsy: an emerging practice model. Physical \& Occupational Therapy in Pediatrics. 1998; 18(1):83-102. doi: 10.1080/j006v18n01_06

[31] Pollock N, Stewart D. Occupational performance needs of school-aged children with physical disabilities in the community. Physical \& Occupational Therapy in Pediatrics. 1998; 18(1):55-68. doi: 10.1080/j006v18n01_04

[32] Soleimani F, Vameghi R, Rassafiani M, Akbar Fahimi N, Nobakht Z. Cerebral Palsy: Motor Types, Gross Motor Function and Associated Disorders. Iranian Rehabilitation Journal. 2011; 9:21-31. 
